University of Wollongong

Research Online

Australian Institute for Innovative Materials -

Papers

Australian Institute for Innovative Materials

$1-1-2015$

A new strategy for achieving a high performance anode for lithium ion batteries-encapsulating germanium nanoparticles in carbon nanoboxes

Dan Li

University of Wollongong, danli@uow.edu.au

Hongqiang Wang

University of Wollongong, hw571@uowmail.edu.au

Hua-Kun Liu

University of Wollongong, hua@uow.edu.au

Zaiping Guo

University of Wollongong, zguo@uow.edu.au

Follow this and additional works at: https://ro.uow.edu.au/aiimpapers

Part of the Engineering Commons, and the Physical Sciences and Mathematics Commons

Research Online is the open access institutional repository for the University of Wollongong. For further information contact the UOW Library: research-pubs@uow.edu.au 


\title{
A new strategy for achieving a high performance anode for lithium ion batteries- encapsulating germanium nanoparticles in carbon nanoboxes
}

\author{
Abstract \\ A novel strategy to improve the electrochemical performance of a germanium anode is proposed via \\ encapsulating germanium nanoparticles in carbon nanoboxes by carbon coating the precursor, \\ germanium dioxide cubes, and then subjecting them to a reduction treatment. The complete and robust \\ carbon boxes are shown to not only provide extra void space for the expansion of germanium \\ nanoparticles after lithium insertion but also offer a large reactive area and reduced distance for the \\ lithium diffusion. Furthermore, the thus-obtained composite, composed of densely stacked carbon \\ nanoboxes encapsulating germanium nanoparticles (germanium at carbon cubes (Ge at CC)), exhibits a \\ high tap density and improved electronic conductivity. Compared to carbon-coated germanium bulks, the \\ Ge at CC material shows excellent electrochemical properties in terms of both rate capability and cycling \\ stability, due to the unique cubic core-shell structure and the effective carbon coating, so that the $\mathrm{Ge}$ at \\ $\mathrm{CC}$ electrode delivers $\approx 497 \mathrm{~mA} \mathrm{~h} \mathrm{g-1}$ at a current rate of $30 \mathrm{C}$ and shows excellent cycling stability of \\ $1065.2 \mathrm{~mA} \mathrm{~h} \mathrm{g-1}$ at $0.5 \mathrm{C}$ for over 500 cycles.
}

\section{Keywords}

carbon, nanoboxes, high, strategy, performance, achieving, anode, lithium, ion, batteries, encapsulating, germanium, nanoparticles

Disciplines

Engineering | Physical Sciences and Mathematics

\section{Publication Details}

Li, D., Wang, H., Liu, H. Kun. \& Guo, Z. (2016). A new strategy for achieving a high performance anode for lithium ion batteries-encapsulating germanium nanoparticles in carbon nanoboxes. Advanced Energy Materials, 6 (5), 1501666-1-1501666-6. 


\section{A New Strategy for Achieving a High Performance Anode for Lithium Ion Batteries - Encapsulating Germanium Nanoparticles in Carbon Nanoboxes}

Dan $\mathrm{Li}^{1.2}$, Hongqiang Wang ${ }^{l}$, Hua Kun Liu ${ }^{l}$, Zaiping Guo ${ }^{1,3^{*}}$

${ }^{1}$ Institute for Superconducting and Electronic Materials, School of Mechanical, Materials and Mechatronics Engineering, University of Wollongong, North Wollongong, NSW 2500, Australia

${ }^{2}$ The College of Chemistry and Molecular Engineering, Zhengzhou University, Zhengzhou, Henan Province 450001, PR China

${ }^{3}$ School of Mechanical, Materials, and Mechatronics Engineering, University of Wollongong, NSW 2500, Australia

E-mail: zguo@uow.edu.au; Tel: +61 242215225 


\begin{abstract}
A novel strategy to improve the electrochemical performance of germanium anode is proposed in this paper via encapsulating germanium nanoparticles in carbon nanoboxes by carbon coating the precursor, germanium dioxide cubes, and then subjecting them to a reduction treatment. The complete and robust carbon boxes are shown to not only provide extra void space for the expansion of germanium nanoparticles after lithium insertion, but also offer a large reactive area and reduced distance for the lithium diffusion. Furthermore, the thus-obtained composite, composed of densely stacked carbon nanoboxes encapsulating germanium nanoparticles (Ge@CC), exhibits a high tap density and improved electronic conductivity. Compared to carbon-coated germanium bulks, the Ge@CC material shows excellent electrochemical properties in terms of both rate capability and cycling stability, due to the unique cubic core-shell structure and the effective carbon coating, so that the Ge@CC electrode delivers about $497 \mathrm{~mA} \mathrm{~h} \mathrm{~g}^{-1}$ at a current rate of $30 \mathrm{C}$ and shows excellent cycling stability of $1065.2 \mathrm{~mA} \mathrm{~h} \mathrm{~g}^{-1}$ at $0.5 \mathrm{C}$ for over 500 cycles.
\end{abstract}

Key words: germanium, carbon boxes, anode, lithium ion batteries

\title{
Introduction
}

Recently, the energy storage community has come under intense pressure to develop lithium ion batteries with high power and energy density, long and stable cycle life, and fast charging-discharging rates in order the satisfy the high demands arising from electric vehicles and portable electronic devices. Germanium is a good candidate anode material for lithium ion batteries due to the great potential of its high specific reversible capacity and outstanding rate capability, as reported in the literature. ${ }^{[1-8]}$ These advantages can be attributed to its high theoretical capacity of $1600 \mathrm{~mA} \mathrm{~h} \mathrm{~g} \mathrm{~g}^{-1}$ (corresponding to $\mathrm{Li}_{4.4} \mathrm{Ge}$ alloy), fast lithium diffusivity ( 400 times greater than in silicon), and high intrinsic electrical conductivity $\left(\sim 100\right.$ times higher than in silicon).$^{[9,10]}$ Nevertheless, like the other group IVA materials (silicon and tin), germanium suffers from huge volume changes (over $300 \%$ after full lithiation) $)^{[11]}$ and particle agglomeration during the alloying/de-alloying process, leading to 
cracking, pulverization, then exfoliation from the current collector, and thus, rapid capacity fading. Therefore, novel synthetic techniques and structural designs to suppress the volume changes, as well as maintaining the conductive network during the charging-discharging process, are the most crucial points for the fabrication of germanium electrode materials with improved cycling stability. In previous studies, germanium-based materials with unique nanostructures, such as nanowires, ${ }^{[12,}{ }^{13]}$ nanotubes, ${ }^{[3]}$ and/or carbonaceous support materials, presented various attractive features as advanced electrode materials for lithium ion batteries. Furthermore, strategies directed towards the synthesis of porous $^{[10]}$ or mesoporous ${ }^{[14]}$ structures have been applied to fully realize the good electrochemical properties of germanium due to the facilitated lithium diffusion and increased active area between the electrode materials and electrolyte. It has been widely accepted that carbon coating is the simplest and most effective approach to improve the electrochemical performance of germanium, which can be ascribed to its effects towards suppressing the volume changes and particle agglomeration, and enhancing electrical conductivity. ${ }^{[9-11,13]}$

A series of germanium-based materials have been synthesized by a facile method of carbon coating and reduction of germanium oxide precursor that was developed in our previous research. ${ }^{[2,15,16]} \mathrm{We}$ found that small particle size, and continuous and robust carbon coatings have significant effects that promote excellent electrochemical properties in germanium anode materials. It is hard, however, to obtain no or less aggregation of germanium nanoparticles without utilizing surfactant templates, which suffer from technological difficulties. ${ }^{[2]}$ Once the nanoparticles are aggregated, the carbon coating strategy is not efficient, since only the superficial nanoparticles can be fully covered. ${ }^{[16]}$ Notably, the aggregation of nanoparticles is usually in the form of random and sparse stacking, which means that the material tends to form loose aggregates after lithiation. The insufficient carbon coating and random aggregates result in fast capacity fading during cycling due to the huge volume expansion of the numerous nanoparticles on the inside of the aggregates after the lithium ion insertion. Furthermore, high tap density is a significant parameter for electrode materials intended for lithium ion batteries with high volumetric energy density. Nanosized materials, however, have intrinsically low tap density due to the loose stacking. ${ }^{[17]}$ Materials composed of densely packed nanosized 
particles, however, promote enhanced tap density and thus higher volumetric energy density. Recently, carbon-coated hollow micro/nanobox materials, such as $\mathrm{CoSnO}_{3} @ \mathrm{C}$ nanoboxes, ${ }^{[18]} \mathrm{Fe}_{2} \mathrm{O}_{3}$ microboxes, ${ }^{[19]}$ and $\mathrm{Fe}_{2} \mathrm{O}_{3} / \mathrm{SnO}_{2}$ microboxes, ${ }^{[20]}$ have drawn considerable attention because their unique cubic structure can tolerate the internal stress generated from the isotropic volume changes, facilitating lithium diffusion and enhanced electronic conductivity. If the micro/nanoboxes are stacked densely, the tap density would be higher than for spheres or random stacking structures.

Inspired by the previous scientific research, we have designed a core-shell structure with germanium nanoparticles encapsulated in intact and robust carbon boxes by covering cubic germanium dioxide with layers of carbon, followed by the reduction of the germanium oxide to germanium particles in the resultant cubic carbon boxes. The synthesis design and unique structure are anticipated to offer multiple advantages for achieving excellent lithium storage performance. First, we used a simple and facile sol-gel reaction to obtain the densely stacked germanium dioxide cubic precursor without any template or multistep synthesis, which provided the basis to obtain the interconnected cubic carbon boxes. Second, the voids and pores formed by the thermal reduction can offer extra space for the volume expansion in the lithiation process. Third, the close-packed carbon boxes not only facilitate the diffusion of lithium ions and electrons, but also limit the formation of the solid electrolyte interphase (SEI) to the external surfaces. Furthermore, the densely packed building blocks (germanium nanoparticles in carbon nanoboxes) ensure better conductivity, higher tap density, and thus, improved volumetric energy density when compared with loose-packed aggregates of germanium nanoparticles coated by carbon layers. The electrochemical performance of our germanium@carbon cubes (Ge@CC) was evaluated, delivering 497 mA h g ${ }^{-1}$ when the C-rate was as high as $30 \mathrm{C}$ and showing excellent cycling stability of $1065.2 \mathrm{~mA} \mathrm{~h} \mathrm{~g}^{-1}$ at $0.5 \mathrm{C}$ for over 500 cycles.

\section{Results and discussion}

The synthesis procedure to prepare the $\mathrm{Ge} @ \mathrm{CC}$ is illustrated in Figure $1 . \mathrm{GeO}_{2}$ cubes were obtained via a facile sol-gel reaction using germanium ethoxide as the germanium source and ammonia as the catalysis agent. ${ }^{[21]}$ It was found that the amount of ammonia plays a significant role in tailoring the 
morphology of the $\mathrm{GeO}_{2}$ (as shown in Figure $\mathrm{S} 1$ in the Supporting Information). An acetylene/argon gas mixture was selected as the carbon source to cover the $\mathrm{GeO}_{2}$ cubes with carbon layers. This strategy relies on the in-situ carbonization reaction of acetylene to form carbon boxes encapsulating the $\mathrm{GeO}_{2}$ cubes, and also simultaneously provide a carbonaceous matrix to prevent the aggregation of the $\mathrm{GeO}_{2}$ cubes. After the reduction treatment via hydrogen, the $\mathrm{GeO}_{2}$ is reduced to metallic germanium nanoparticles, which are completely encapsulated in the carbon boxes. Importantly, the voids left due to the reduction from $\mathrm{GeO}_{2}$ to germanium provide space for the volume expansion of the germanium nanoparticles in the lithiation process, thus efficiently suppressing cracking of the electrode material. The voids and pores can also accommodate electrolyte, which allows fast transport of lithium ions during the charging-discharging process. Furthermore, the carbon nanoboxes are interconnected with each other to form a continuous conductive framework, facilitating the transfer of electrons. In contrast, the carbon shells of Ge@CB can only cover the external germanium nanoparticles of the aggregated bulks to some extent due to the large size of these aggregates (shown in Figure S1). After several cycles of charge/discharge, these fragile carbon shells in Ge@CB would break because of the huge volume expansion after lithiation of the internal uncoated germanium nanoparticles, resulting in cracking, and eventually, pulverization of the electrode.

The crystal structure of the $\mathrm{GeO}_{2}$ precursor, carbon coated intermediates, and germanium@carbon (Ge@C) products were determined by X-ray diffraction (XRD), as shown in Figure 2(a) and Figure S2. All the diffraction peaks of the $\mathrm{GeO}_{2}$ precursor and the carbon coated intermediates can be well indexed to the hexagonal phase of $\mathrm{GeO}_{2}$ (JCPDS card no. 36-1463). Although the carbonization of acetylene gas has a reductive nature, there is no peak that can be attributed to germanium, as shown in the XRD patterns of intermediates. Therefore, the carbon contents of the Ge@C products can be determined through thermogravimetric analysis (TGA) of the $\mathrm{GeO}_{2} @ \mathrm{C}$ intermediates (as shown in Figure S3), and the calculated carbon values are 4.5\% and 7.6\% for $\mathrm{Ge} @ \mathrm{CB}$ and $\mathrm{Ge} @ \mathrm{CC}$, respectively. After the reduction treatment, the $\mathrm{GeO}_{2}$ can be completely converted to metallic germanium, corresponding to the diamond cubic phase (JCPDS card no. 40-0545). From the XRD patterns of both Ge@C products, no carbon peaks can be detected, which may be due to the 
disordered nature of carbon and the high intensity of the (111) peak of germanium. The Raman spectra of the $\mathrm{GeO}_{2}$ cubes and $\mathrm{Ge} @ \mathrm{CC}$ are shown in Figure 2(b) and indicate their chemical bonding. For the $\mathrm{GeO}_{2}$ cube precursor, the peaks can be related to the vibrations from $\mathrm{GeO}_{2}$, which match well with previous reports in the literature. ${ }^{[22]}$ After the carbon-coating and reduction treatments, a peak appears at $300 \mathrm{~cm}^{-1}$, which can be attributed to the optical mode of metallic germanium. Two prominent peaks are also located at $1332 \mathrm{~cm}^{-1}$ and $1602 \mathrm{~cm}^{-1}$, corresponding to the $\mathrm{D}$ band and $\mathrm{G}$ band of the carbon boxes, respectively.

The structural and morphological characterizations were conducted through field emission scanning electron microscopy (FE-SEM) and transmission electron microscopy (TEM) observations. From the low magnification FE-SEM image shown in Figure 3(a), it can be observed that the obtained Ge@CC composite clearly inherits the uniform shape and size of its particles from the $\mathrm{GeO}_{2}$ cubes, with the coated carbon shells also displaying a cubic shape. These germanium nanoparticles encapsulated in carbon boxes are closely packed with a higher tap density of $1.13 \mathrm{~g} \mathrm{~cm}^{-3}$ compared to that of the carbon coated germanium bulks $\left(0.81 \mathrm{~g} \mathrm{~cm}^{-3}\right)$. After the reduction treatment by hydrogen/argon gas, voids and spaces were generated in the carbon nanoboxes. There are clearly distinct hollow cavities in the central and edge regions of a Ge@CC particle, as shown by the high magnification FE-SEM image (Figure 3(b)), which can provide space to accommodate the volume changes during the lithiation/de-lithiation process. The TEM images gave more specific details on the carbon boxes. Figure 3(c) reveals the continuous and complete nature of the carbon shells, which are about $30 \mathrm{~nm}$ in thickness, and can protect the integrity of the core-shell structure and inhibit the continual formation of a solid electrolyte interphase layer. ${ }^{[23]}$ The elemental mapping images (Figure 3(e) and (f)) and energy dispersive X-ray spectroscopy (EDXS) line profiles (Figure 3(g)) further demonstrate the morphology and distribution of germanium within the carbon box.

To determine the functionality of the effective core-shell structure with respect to the electrochemical properties, cycling stability and rate capability tests were carried out. Figure 4(a) shows the cycling performance of the $\mathrm{Ge} @ \mathrm{CC}$ and $\mathrm{Ge} @ \mathrm{CB}$ composites at $0.2 \mathrm{C}$ for the first 5 cycles and at $0.5 \mathrm{C}$ for the remaining cycles. The $1 \mathrm{C}$ rate is defined as current density of $1600 \mathrm{~mA} \mathrm{~g}^{-1}$. Remarkably, the Ge@CC 
exhibits outstanding and stable cyclability. No capacity fading is observed during cycling. After 500 cycles, the electrode still delivers a reversible capacity of $1065.2 \mathrm{~mA} \mathrm{~h} \mathrm{~g}^{-1}$, corresponding to $91.4 \%$ of the capacity at the $10^{\text {th }}$ cycle. In contrast, the Ge@CB electrode undergoes a gradual capacity decay to a capacity of $905.6 \mathrm{~mA} \mathrm{~h} \mathrm{~g}^{-1}$ at the $200^{\text {th }}$ cycle, corresponding to $82 \%$ of the capacity at the $10^{\text {th }}$ cycle. The structure of Ge@CC is maintained after 500 discharge/charge cycles (as shown in Figure S9), indicating that the cubic carbon shell can withstand the volume changes during the alloying/dealloying process and thus retain its structural stability. The Ge@CC was further investigated by rate capacity testing from $0.1 \mathrm{C}$ to $30 \mathrm{C}$, as shown in Figure 4(b). The high capacities in the first two cycles can be attributed to the large density of active sites in or between the carbon box and the germanium nanoparticles, and in the grain boundary areas of germanium nanoparticles. ${ }^{[16,24]}$ The discharge capacity was $1235.5 \mathrm{~mA} \mathrm{~h} \mathrm{~g}^{-1}$ at the $5^{\text {th }}$ cycle at $0.1 \mathrm{C}$. The average capacities were 1128.1 $\mathrm{mA} \mathrm{h} \mathrm{g}{ }^{-1}$ at $1 \mathrm{C}, 1050 \mathrm{~mA} \mathrm{~h} \mathrm{~g}^{-1}$ at $5 \mathrm{C}, 970.9 \mathrm{~mA} \mathrm{~h} \mathrm{~g}^{-1}$ at $10 \mathrm{C}, 847.1 \mathrm{~mA} \mathrm{~h} \mathrm{~g}^{-1}$ at $15 \mathrm{C}$, and $742.1 \mathrm{~mA}$ $\mathrm{h} \mathrm{g}^{-1}$ at $20 \mathrm{C}$. Even at the high current rate of $30 \mathrm{C}$, the reversible capacity of Ge@CC reaches an average value of $615.3 \mathrm{~mA} \mathrm{~h} \mathrm{~g}^{-1}$. Notably, there is increasing capacity during cycling in both the Ge@CB and the Ge@CC samples, which can be ascribed to an electrochemical activation process. The capacity also recovers to $1210 \mathrm{~mA} \mathrm{~h} \mathrm{~g}^{-1}$ when the current rate returns to $0.1 \mathrm{C}$, indicating good reversibility. In the case of the Ge@CB sample, the rate capacity is inferior compared to the $\mathrm{Ge} @ \mathrm{CC}$, delivering an average capacity of only $497 \mathrm{~mA} \mathrm{~h} \mathrm{~g}^{-1}$ at $30 \mathrm{C}$. The discharge/charge voltage profiles of Ge@CC are shown in Figure 4(c) in the range of 0.01-1.5 V at different rates. The Ge@CC presents distinct voltage plateaus at about $0.2 \mathrm{~V}$ in the discharge profile and $0.5 \mathrm{~V}$ in the charge profile, corresponding to the alloying and de-alloying of Li-Ge, respectively. The Ge@CC delivered the third discharge capacity of $1238.4 \mathrm{~mA} \mathrm{~h} \mathrm{~g}^{-1}$ and the third charge capacity of $1100 \mathrm{~mA} \mathrm{~h} \mathrm{~g}^{-1}$ at $0.1 \mathrm{C}$ with a coulombic efficiency of $88.8 \%$, while the Ge@CB presented the third discharge capacity of 1349.8 $\mathrm{mA} \mathrm{h} \mathrm{g}{ }^{-1}$ and the third charge capacity of $1131.3 \mathrm{~mA} \mathrm{~h} \mathrm{~g}^{-1}$ at $0.2 \mathrm{C}$, indicating a coulombic efficiency of $83.8 \%$. To evaluate the toughness of carbon nanoboxes, a series of ex-situ TEM measurements were performed at different charge voltages as shown in Figure S10. The intact carbon nanoboxes after fully lithiation indicates that the voids can provide enough space to accommodate the volume 
expansion for germanium nanoparticles and this kind of carbon shell are strong enough to suppress the volume expansion of germanium nanoparticles.

The lithium storage mechanism of Ge@CC composite was further evaluated through cyclic voltammetry $(\mathrm{CV})$ in a voltage window of 0-3.0 V, and the profiles are shown in Figure 4(d). In the first reduction cycle, the two peaks located at 1.3 and $1.0 \mathrm{~V}$ can be ascribed to the irreversible reactions of lithium and functional groups on the carbon surface, ${ }^{[25]}$ and the formation of the solid electrolyte interphase (SEI), respectively. Both types of irreversible lithium storage contribute to the high specific capacity of the initial cycle and are consistent with the cycling and rate performance. There are four peaks detected in the range between 0.7 and $0.1 \mathrm{~V}$, which can be related to the different $\mathrm{Li}_{x} \mathrm{Ge}$ alloys formed via lithium alloying reactions, ${ }^{[2,13,26]}$ while the peaks between 0.25 and $0.75 \mathrm{~V}$ in the oxidation scan can be associated with the de-alloying reactions, where lithium is extracted from the Li-Ge alloys to eventually form germanium.

The unique cubic core-shell structures contribute to the excellent electrochemical properties of $\mathrm{Ge} @ \mathrm{CC}$ composite. First, the obtained precursor, the $\mathrm{GeO}_{2}$ cubes, promote the formation of robust and complete carbon boxes that act as shells, which can maintain the integrity of the materials by tolerating the volume changes during the lithium insertion/extraction process. In contrast, the carbon shells for the Ge@CB sample are only limited to the outer layers of the germanium bulks, which are too large for their interior particles to be uniformly coated by carbon. This kind of carbon shell is too fragile and brittle to withstand the huge volume changes. Furthermore, the germanium nanoparticles encapsulated in the carbon box building blocks ( $300 \mathrm{~nm}$ in size $)$ can be densely packed together to improve the tap density of the composite, which can further increase the volumetric density. Importantly, the voids and spaces formed because of the reduction from $\mathrm{GeO}_{2}$ to germanium can accommodate the volume expansion during the lithiation reaction, enabling this composite to further withstand the repeated cycling. In addition, the cubic carbon shells act as an electrically conductive network to facilitate the transfer of electrons and lithium ions, which guarantees the high electrical conductivity of the electrode material. 


\section{Conclusion}

In summary, we have developed a simple and surfactant-free method to obtain uniform $\mathrm{GeO}_{2}$ cubes and then cover their surfaces with carbon layers, and the carbon-coated germanium oxide cubes are then converted to cubic Ge@CC core-shell structures after a reduction treatment. The obtained robust and complete carbon nanoboxes form an interconnected network, and the voids in the carbon nanoboxes can effectively buffer the volume changes during lithiation/de-lithiation and protect the germanium nanoparticles from cracking and/or crumbling, so that the integrity of the composite is maintained. Furthermore, the dense stacking of the germanium nanoparticles encapsulated in the carbon boxes gives the composite a high tap density. The unique structure endows the material with improved specific capacity, superior rate capability, and very good cycling performance.

\section{Acknowledgments}

Financial support provided by the Australian Research Council (ARC) through an ARC Discovery project (DP1094261) is gratefully acknowledged. Moreover, the authors would like to thank Dr Tania Silver for critical reading of the manuscript and valuable remarks.

\section{References}

[1] P. G. Bruce, B. Scrosati, J.-M. Tarascon, Angew. Chem. Int. Ed. 2008, 47, 2930.

[2] K. H. Seng, M.-H. Park, Z. P. Guo, H. K. Liu, J. Cho, Angew. Chem. Int. Ed. 2012, 51, 5657.

[3] T. Song, H. Cheng, H. Choi, J.-H. Lee, H. Han, D. H. Lee, D. S. Yoo, M.-S. Kwon, J.-M. Choi, S. G. Doo, H. Chang, J. Xiao, Y. Huang, W. I. Park, Y.-C. Chung, H. Kim, J. A. Rogers, U. Paik, ACS Nano 2011, 6, 303.

[4] T. Kennedy, M. Bezuidenhout, K. Palaniappan, K. Stokes, M. Brandon, K. M. Ryan, ACS Nano 2015, 9, 7456.

[5] H. Kim, Y. Son, C. Park, M.-J. Lee, M. Hong, J. Kim, M. Lee, J. Cho, H. C. Choi, Nano Lett. $2015,15,4135$.

[6] X. Li, Z. Yang, Y. Fu, L. Qiao, D. Li, H. Yue, D. He, ACS Nano 2015, 9, 1858. 
[7] W. Tang, Y. Liu, C. Peng, M. Y. Hu, X. Deng, M. Lin, J. Z. Hu, K. P. Loh, J. Am. Chem. Soc. 2015, 137, 2600.

[8] M. I. Bodnarchuk, K. V. Kravchyk, F. Krumeich, S. Wang, M. V. Kovalenko, ACS Nano 2015, 8, 2360.

[9] H. Lee, J. Cho, Nano Lett. 2007, 7, 2638.

[10] M.-H. Park, K. Kim, J. Kim, J. Cho, Adv. Mater. 2010, 22, 415.

[11] a) J. Cheng, J. Du, CrystEngComm 2012, 14, 397; b) A. M. Chockla, M. G. Panthani, V. C. Holmberg, C. M. Hessel, D. K. Reid, T. D. Bogart, J. T. Harris, C. B. Mullins, B. A. Korgel, J. Phys. Chem. C 2012, 116, 11917.

[12] a) F.-W. Yuan, H.-J. Yang, H.-Y. Tuan, ACS Nano 2012, 6, 9932; b) C. K. Chan, X. F. Zhang, Y. Cui, Nano Lett. 2007, 8, 307; c) X. H. Liu, S. Huang, S. T. Picraux, J. Li, T. Zhu, J. Y. Huang, Nano Lett. 2011, 11, 3991.

[13] M.-H. Seo, M. Park, K. T. Lee, K. Kim, J. Kim, J. Cho, Energy Environ. Sci. 2011, 4, 425.

[14] L. C. Yang, Q. S. Gao, L. Li, Y. Tang, Y. P. Wu, Electrochem. Commun. 2010, 12, 418.

[15] a) D. Li, C. Feng, H. k. Liu, Z. Guo, J. Mater. Chem. A 2015, 3, 978; b) K. H. Seng, M.-h. Park, Z. P. Guo, H. K. Liu, J. Cho, Nano Lett. 2013, 13, 1230.

[16] L. Li, K. H. Seng, C. Feng, H. Liu, Z. Guo, J. Mater. Chem. A 2013, 1, 7666.

[17] Z. Chen, J. R. Dahn, J. Electrochem. Soc. 2002, 149, A1184.

[18] Z. Wang, Z. Wang, W. Liu, W. Xiao, X. W. Lou, Energy Environ. Sci. 2013, 6, 87.

[19] L. Zhang, H. B. Wu, S. Madhavi, H. H. Hng, X. W. Lou, J. Am. Chem. Soc. 2012, 134, 17388.

[20] L. Zhang, H. B. Wu, X. W. Lou, J. Am. Chem. Soc. 2013, 135, 10664.

[21] M. Javadi, Z. Yang, J. G. C. Veinot, Chem. Commun. 2014, 50, 6101.

[22] G. Cui, L. Gu, L. Zhi, N. Kaskhedikar, P. A. van Aken, K. Müllen, J. Maier, Adv. Mater. 2008, 20, 3079; b) P. Benzi, E. Bottizzo, C. Demaria, G. Infante, G. Iucci, G. Polzonetti, J. Appl. Phys. 2007, 101, 124906.

[23] N. Liu, H. Wu, M. T. McDowell, Y. Yao, C. Wang, Y. Cui, Nano Lett. 2012, 12, 3315.

[24] Z.-S. Wu, W. Ren, L. Wen, L. Gao, J. Zhao, Z. Chen, G. Zhou, F. Li, H.-M. Cheng, ACS Nano 2011, 4, 3187. 
[25] a) K. Tang, R. J. White, X. Mu, M.-M. Titirici, P. A. Aan Aken, J. Maier, ChemSusChem 2012, 5, 400; b) 2012, 5, 400; Y. Matsumura, S. Wang, J. Mondori, J. Electrochem. Soc. 1995, 142, 2914.

[26] a) H. Lee, M. G. Kim, C. H. Choi, Y.-K. Sun, C. S. Yoon, J. Cho, J. Phys. Chem. B 2005, 109, 20719; b) S. Yoon, C-M Park, H-J Sohn, Electrochem. Solid-State Lett. 2008, 11, A42. 


\section{Figures and Captions}

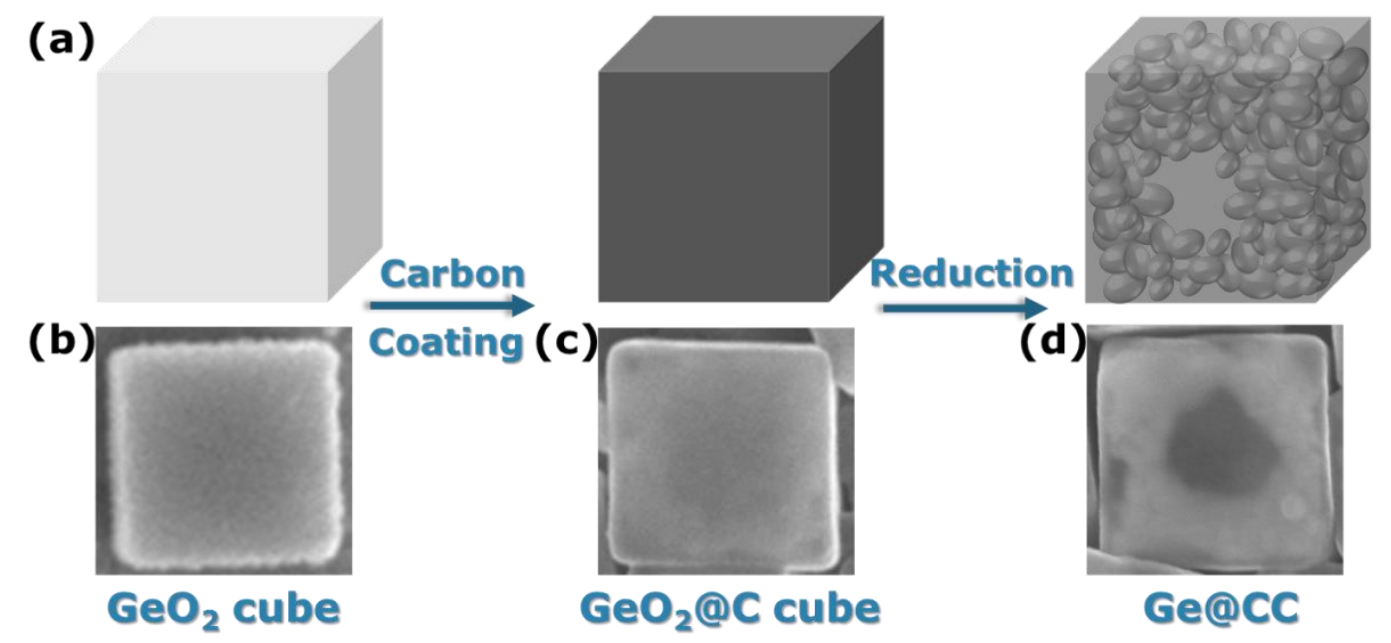

Figure 1. (a) Schematic illustration of the formation process for Ge@CC. Scanning electron microscope (SEM) images of (b) $\mathrm{GeO}_{2}$ cube precursor, (c) $\mathrm{GeO}_{2} @ \mathrm{C}$ cube, (d) $\mathrm{Ge} @ \mathrm{CC}$. The carbon coated $\mathrm{GeO}_{2}$ cube intermediate is achieved by the thermal decomposition of acetylene/argon gas, and then is reduced by hydrogen/argon gas to obtain the $\mathrm{Ge} @ \mathrm{CC}$. 

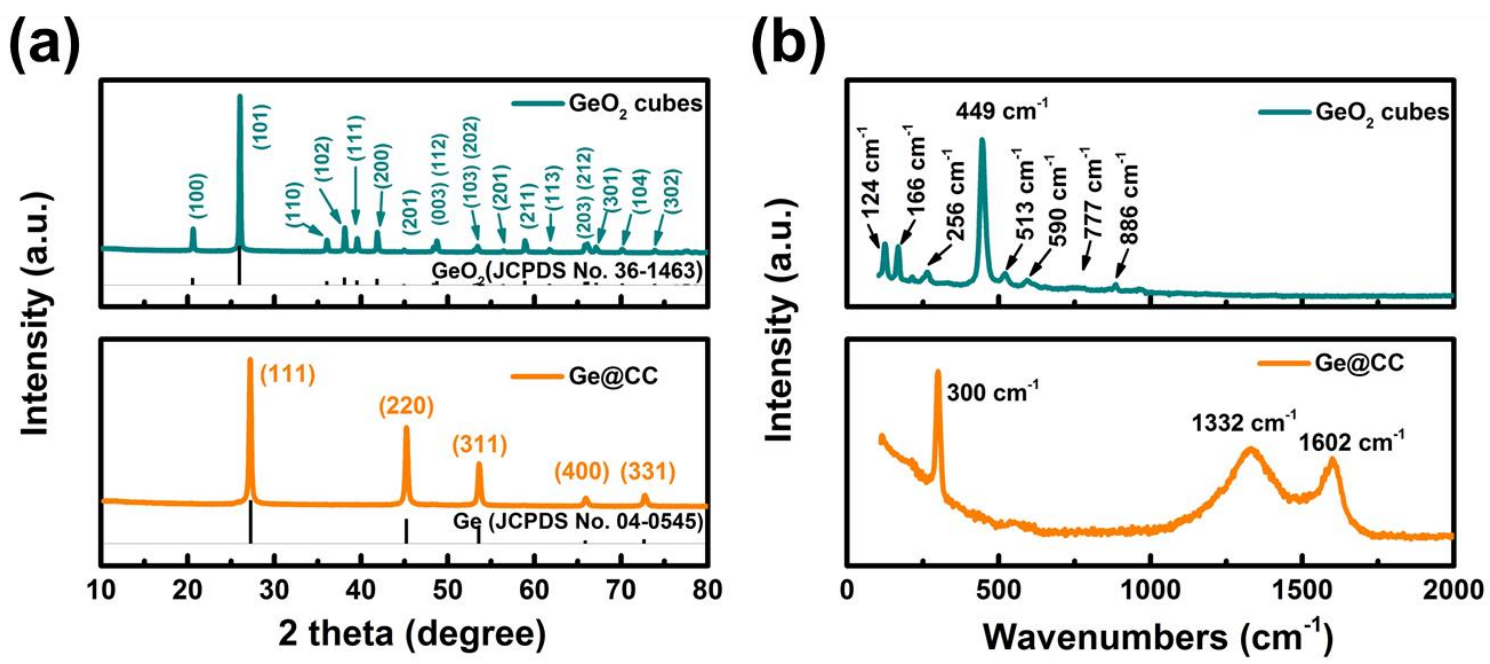

Figure 2. (a) Powder X-ray diffraction patterns and (b) Raman spectra of $\mathrm{GeO}_{2}$ cube and $\mathrm{Ge} @ \mathrm{CC}$. 


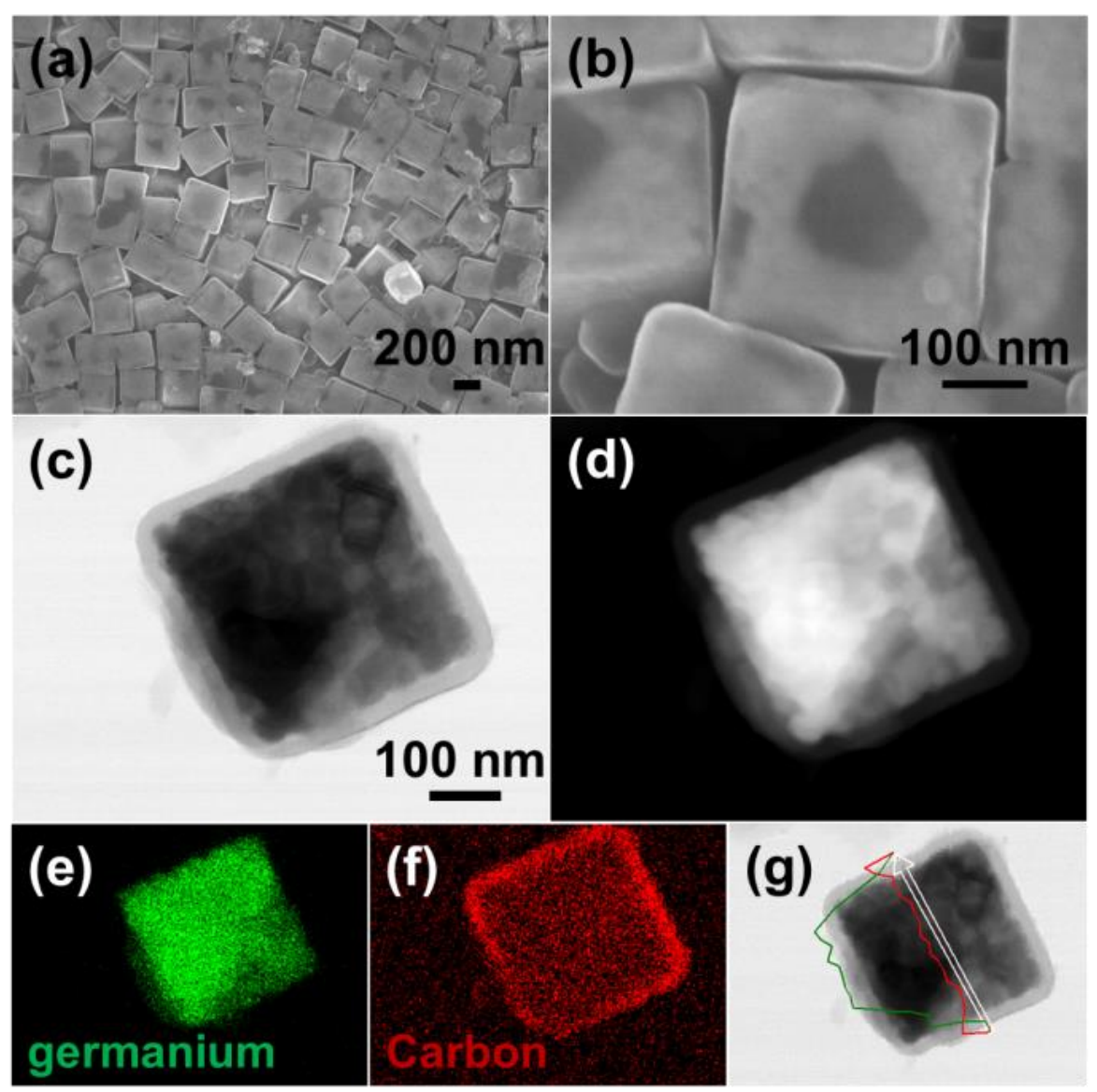

Figure 3. (a, b) SEM images of Ge@CC, c) bright field and (d) dark field images of Ge@CC collected by scanning transmission electron microscopy (STEM); images of the distributions of (e) germanium, (f) carbon, and (g) the corresponding line profile from the elemental analysis. 
(a)

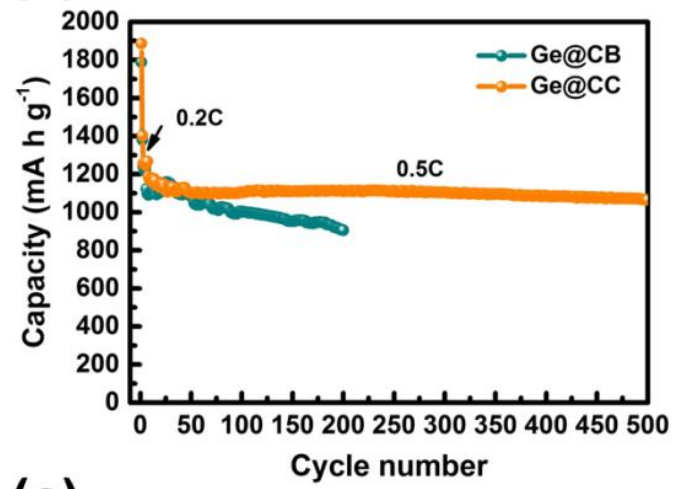

(c)

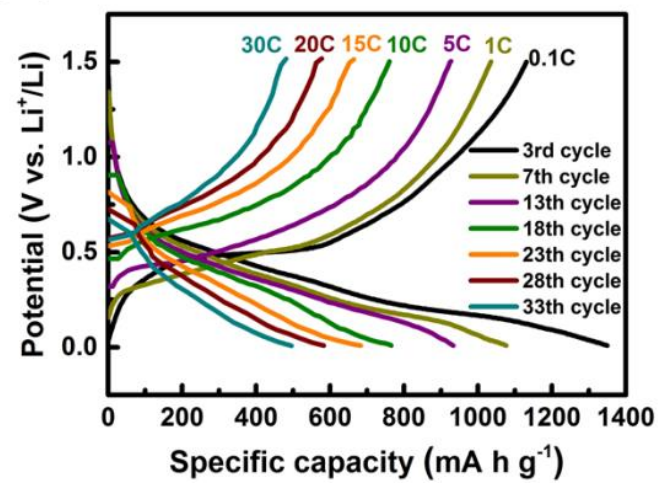

(b)

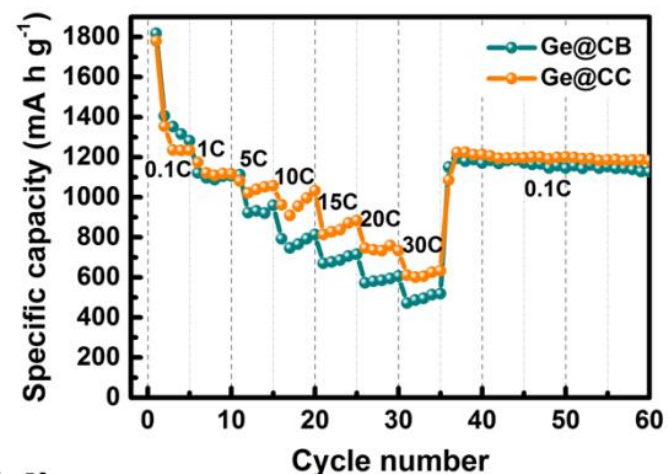

(d)

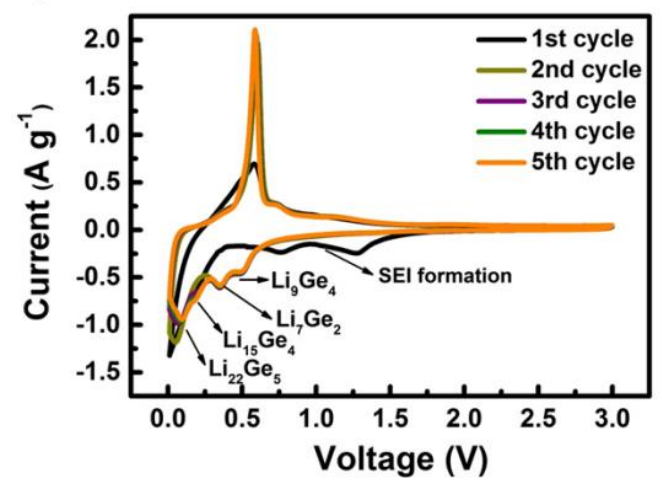

Figure 4. (a) Cycling performance of $\mathrm{Ge} @ \mathrm{CB}$ and $\mathrm{Ge} @ \mathrm{CC}$ at $0.5 \mathrm{C}$ current rate $(0.2 \mathrm{C}$ current rate was applied in the first 5 cycles in order to activate the electrode). (b) Comparison of rate capability of Ge@CB and Ge@CC at various current rates from 0.1 C-30 C. (c) Galvanostatic charge/discharge profiles for selected cycles at different current rates from $0.1 \mathrm{C}-30 \mathrm{C}$ (corresponding to (b)). (d) Cyclic voltammetry profiles of Ge@CC at the scan rate of $0.1 \mathrm{mV} \mathrm{s}^{-1}$ for 5 cycles. 